\title{
SERIES INTRODUCTION \\ The expanding network of redox signaling: new observations, complexities, and perspectives
}

\author{
Roy J. Soberman \\ Renal Unit and Department of Medicine, Massachusetts General Hospital, Harvard Medical School, Charlestown, Massachusetts, USA \\ J. Clin. Invest. 111:571-574 (2003). doi:10.1172/JCI200318099.
}

Over thirty years ago, the observations that eventually led to the discovery of the NADPH oxidase were made by Baehner, Karnovsky, and colleagues (1-3). These have served as a focal point of interest in placing reactive oxygen species (ROS) in the conceptual forefront of the biomedical community. Over the last decade, the examination of the roles of oxygen and redox tone in regulating cell function has turned inward to the intracellular environment. Because oxidative metabolism is central to the biology and health of all humans, how we respond to conditions of low and high oxygen stress has become a critical consideration in biology and medicine. Humans live in a world where we continually balance the use of oxygen as a source of energy, and as a source of cellular injury. The generation of oxygen radicals secondary to mitochondrial disruption, the activation of cellular NADPH oxidases, the metabolism of xenobiotics, or other forms of oxidative stress can lead to mutations in DNA, lipid peroxidation, and protein damage. We have therefore evolved a marvelously complex system of both defense mechanisms and sensing mechanisms for changes in cellular redox tone. These include the enzymes superoxide dismutase, catalase, and glutathione peroxidase that detoxify ROS. We have also developed signaling mechanisms that utilize ROS to initiate processes that allow cells to survive exposure to oxidative stress within certain tolerances, but also, when stress and damage become too great, to ensure cell death. How these pathways are initiated and controlled on a molecular basis by ROS and also molecular oxygen is at the heart of what is generally considered redox signaling and the response to oxidative

\footnotetext{
Address correspondence to: Roy J. Soberman, Renal Unit and Department of Medicine, Massachusetts General Hospital, Harvard Medical School, Building 149, Charlestown Navy Yard, 13th Street, Charlestown, Massachusetts 02129, USA. Phone: (617) 726-3747; Fax: (617) 726-5669;

E-mail: Soberman@helix.mgh.harvard.edu.

Conflict of interest: The author has declared that no conflict of interest exists.

Nonstandard abbreviations used: reactive oxygen species (ROS); hydrogen peroxide $\left(\mathrm{H}_{2} \mathrm{O}_{2}\right)$; hypoxia inducible factor 1 (HIF-1); carboxy-terminal binding protein (CtBP); apoptosis-inducing factor (AIF); apoptosis signal-regulating kinase 1 (ASK1).
}

stress. The molecular species that fall under the term ROS include superoxide, hydrogen peroxide $\left(\mathrm{H}_{2} \mathrm{O}_{2}\right)$, hydroxyl radical, and singlet oxygen. Each of these can play a role in a variety of intracellular processes. Finally, we have adapted these molecular species, particularly $\mathrm{H}_{2} \mathrm{O}_{2}$ and $\mathrm{NO}$, as signaling molecules in multiple biological processes.

The organization of redox signaling and the use of oxygen to transmit information are proving to be much more complex than one could have originally imagined. As this Perspective series evolved, discussions with the authors suggested that we should cast a wide net and include various mechanisms by which oxygen and reducing equivalents have been adapted to transmit information within cells, or to create cellular damage. Our discussions seemed to raise as many questions as they answered. In the resulting series, several broad areas emerge that may focus the thinking of readers in new directions.

\section{Redox-responsive and oxygen-sensitive transcription}

Sensing nuclear oxygen tension. Since its original description, the study of the transcription factor hypoxia inducible factor 1 (HIF-1) has demonstrated its central role in regulating the body's response to changing oxygen levels (4-6). It is the paradigm for how molecular oxygen can regulate transcription. The functions of HIF-1 target genes have been divided into categories that include cell proliferation and viability, erythropoiesis and iron metabolism, and vascular development and remodeling (6), and the expression of these genes is induced when oxygen tension dips below a safe level. To prevent the continuous overexpression of these genes, cells utilize prolyl hydroxylases (7-9) to hydroxylate HIF- $1 \alpha$. Hydroxylation targets HIF- $1 \alpha$ for binding to the von Hippel-Lindau protein ( $\mathrm{pVHL}$ ), which is the recognition component of an E3 ubiquitin protein ligase, and ubiquitination of HIF- $1 \alpha$. This results in the targeting of HIF-1 $\alpha$ for destruction by the proteasome (4-9). Cells lacking functional pVHL cannot degrade HIF and thus overproduce mRNAs encoded by HIF target genes. Oxygenation of asparagine also blocks the recruitment of coactivating proteins (10). The functional inactivation of the von Hippel-Lindau 
tumor suppressor is observed in hemangioblastomas and in clear cell renal carcinoma (4-9). Thus, oxygen, per se, functions as a negative regulator of transcription. This topic will be addressed in the Perspective by Michal Safran and William Kaelin (11).

NADs as multifunctional redox sensors. A recently described mechanism for redox-based transcription involves the use of the NAD cofactors $\mathrm{NADH}, \mathrm{NAD}^{+}$, $\mathrm{NADPH}$, and $\mathrm{NADP}^{+}$as regulators of genes based on their redox state (12-14). This paradigm is exemplified by the transcription factors Clock:BMAL1 and NPAS2:BMAL1 $(12,13)$. These are heterodimeric transcription factors that control gene expression as a function of the light-dark cycle. The binding of these transcription factors, and presumably transcription efficiency, are markedly enhanced by $\mathrm{NADH}$ and $\mathrm{NADPH}$, but inhibited by $\mathrm{NAD}^{+}$and $\mathrm{NADP}^{+}$. In these cases, they function as allosteric modifiers, where the effectiveness of NADs does not depend on modification of proteins. This form of redox control has also been proposed for the carboxy-terminal binding protein (CtBP) (14), which can function as a cofactor for repression of transcription. Its action is dependent on adenine dinucleotide cofactors and has been suggested in certain circumstances to be related to the oxidation/reduction state of these molecules, with NADH being 100- to 1,000-fold more effective than $\mathrm{NAD}^{+}$. This would allow the ratio of nuclear NADH to NAD ${ }^{+}$ to function as a redox sensor for transcription. The number of transcription factors and genes regulated in this manner is not known, but the potential for this mechanism to be employed in settings such as inflammation and cell growth is apparent.

Direct modification of transcription factors by ROS. Direct modification of transcription factors by ROS is exemplified by the Escherichia coli transcription factor OxyR $(15,16)$. In the case of OxyR, the reduced form of the protein can bind to DNA but cannot induce transcription. Selective cysteines undergo posttranslational modification, leading to the activation of transcription. These modifications result in the formation of S-NO, $S$-OH, and $S$-glutathionyl adducts. These modified forms of OxyR are transcriptionally active but differ in structure, cooperative properties, DNA-binding affinity, and promoter activities.

\section{Eicosanoid-based signaling}

The use of arachidonic acid as a template for the enzymatic insertion of oxygen (17-19) exemplifies how the body uses oxygen to impart specific signaling information. As described in a previous JCI Perspective series (20-27), the numbers and function of oxygenated derivatives of arachidonic acid vary widely. These were originally described as including the cyclooxygenase derivatives, the prostaglandins, the thromboxanes, and the prostacyclins. This list was expanded to include leukotrienes and then products of oxidation of 12-, 15-, and 8-lipoxygenases, as well as cytochrome P450s. More recently described products such as isoprostanes and products formed by multiple lipoxygenases (lipoxins) have been shown to be formed during periods of cellular stress or damage. The critical difference in the biological properties of each of these molecules is the number, position, and stereochemistry of oxygen insertions, which then determine the geometry and information content of each molecule. Thus, in the world of eicosanoids, oxygen is the currency of information. How cells and tissues regulate the balance of each of these oxidations, and the biological properties of these molecules, will remain a rewarding area of investigation for the foreseeable future and will be addressed by the author and Peter Christmas in this Perspective series (28).

\section{Sensing and harnessing ROS}

The role of multifunctional proteins in redox signaling. One of the most intriguing aspects of redox signaling and oxidative stress is that proteins involved in the transduction of cellular redox changes have recently been shown to be multifunctional. This is illustrated by apoptosis-inducing factor (AIF). The cell biology of this protein and its relation to disease are just being unraveled, but AIF illustrates how single proteins that control oxidation and reduction events can have distinct functions that are determined by different intracellular microenvironments and contexts. AIF can function as an NADH oxidase in mitochondria, and potentially as a scaffolding protein for caspase-independent cell death in the nucleus $(29,30)$. The harlequin $(H q)$ mouse displays progressive degeneration of cerebellar and retinal neurons with aging (29). A proviral insertion in the first intron of the Aif gene results in an $80 \%$ decrease in AIF expression. Work by Klein, Ackerman, and colleagues (30) using these mice has led to the proposal that AIF can act as a mitochondrial free radical scavenger. In this series Susan Ackerman and Jeffrey Klein will focus on the role of AIF in oxidative stress and neurodegeneration (31). An alternate proposal, based on these studies, suggests that AIF could function in the electron transport chain (29). Genetic deletion of AIF would lead to uncoupling of mitochondrial electron flow with a concomitant increase in ROS, mitochondrial membrane disruption, and trafficking of AIF to the nucleus, where it serves as a scaffold for caspase-independent cell death. The redox and scaffolding functions are carried out by different parts of the AIF molecule.

The CtBP protein has been shown to be multifunctional as well. Besides functioning as a cofactor for transcription by sensing the ratio of $\mathrm{NADH}$ to $\mathrm{NAD}^{+}$, it has recently been shown to function as a short-chain dehydrogenase (32), raising the possibility that a combination of a substrate and the nuclear redox state can determine its function. Whether and how these functions are integrated is unknown. It is intriguing to speculate that an endogenous dehydrogenase substrate could impact the function of NAD cofactors to regulate the corepressor function of CtBP directly, or indirectly secondary to the catalytic use of adenine dinucleotide cofactors.

Mechanisms of cellular damage. The damage that is wreaked on cells as a byproduct of oxidative stress and the generation of ROS and other electrophiles can 
obviously be lethal. The reactive properties of secondgeneration molecules lead to cellular damage that includes mutations of DNA, damage to and inactivation of proteins including $\mathrm{p} 53$, and lipid peroxidation $(33,34)$. These mechanisms are reviewed in this Perspective series by Lawrence Marnett, James Riggins, and James West (33). The consequences and mechanisms of these reactions remain an area of intense scrutiny.

Surprisingly, these lethal effects of excessive ROS and electrophiles can also be harnessed productively. The generation of ROS by expanding populations of antigen-specific $\mathrm{T}$ cells is responsible for limiting the size of the population of these clones within the body. The mechanisms by which this occurs are discussed by Hildeman et al. in this series (35) and demonstrate how these molecules can be used to modulate processes. The use of ROS to modulate specific intracellular functions leads to challenge of the overall cellular organization of redox signaling.

\section{The cellular organization of redox signaling and the pivotal role of cysteine}

Perhaps the greatest overall challenge is to understand the strategy that underlies the organization of redox signaling in cells - if such a strategy exists. A critical unresolved question is to what extent ROS are generated within a microenvironment and act locally, and to what extent they originate from sources such as cellular NADPH oxidases, nitric oxide synthases, or mitochondria, diffuse widely, and then have their signaling integrated by proteins such as thioredoxins and other redoxins. This question is addressed by Carl Nathan in this series (36). It is likely that both mechanisms are operative. The role of cysteines in the transduction of redox signals deserves special mention and is described below.

Hydrogen peroxide is well recognized as an intracellular signaling molecule (37). $\mathrm{H}_{2} \mathrm{O}_{2}$ has been shown to be important for the transduction of signals by PDGF (38), insulin (39), leukotriene $B_{4}(40)$, and TNF- $\alpha$ (37). It functions by oxidizing cysteines; however, the consequences of cysteine oxidation depend on the molecular context and acidic $\mathrm{p} K_{\mathrm{a}}$ (acid constant) of the specific cysteine being oxidized (the $\mathrm{p} K_{\mathrm{a}}$ of most cysteines is alkaline). As reviewed by Rhee et al. (37), the sequence Cys-X-X-Gly-X-X-Arg-Ser/Thr is a signature motif in certain protein tyrosine phosphatases. The cysteine $\left(\mathrm{p} K_{\mathrm{a}}\right.$ 4.7-5.4) exists as a thiolate anion at neutral $\mathrm{pH}$ (41) and forms a thiol-phosphate intermediate in the catalytic mechanism of protein tyrosine phosphatases. The reversible oxidation of this cysteine inactivates protein tyrosine phosphatases, resulting in unopposed phosphorylation and proliferation $(42,43)$.

A second critical motif that has been identified in signaling by ROS is the Cys-X-X-Cys motif. This is a signature motif for cellular redoxins, including thioredoxin, glutaredoxin, and nucleoredoxin. It is also present in members of the PP1 subfamily of protein phosphatases (44), and in protein disulfide isomerase. The best-known example of these molecules is thioredoxin. Thioredoxin functions to reduce selected proteins. Thioredoxin is also associated with apoptosis signal-regulating kinase 1 (ASK1) $(45,46)$. In response to signaling by TNF- $\alpha$, ROS are generated that eventually lead to the oxidation of the core cysteines of thioredoxin. The oxidation of thioredoxin leads to a conformational change, resulting in its dissociation from ASK1. ASK1 is a MAP kinase kinase kinase, and its release from thioredoxin allows it to mediate stress signaling by TNF- $\alpha$ through the activation of stress-activating protein kinases and p38 kinases. The full extent of the networks and proteins that are regulated by redoxins remains to be determined. The sequencing of the human genome has the potential to identify many unappreciated proteins that share motifs such as those of phosphatases and thioredoxin. The oxidation of cysteines has also been implicated in the processing of antigen $(47,48)$. The oxidation of Cys118 of Ras results in activation, rather than inhibition, of Ras resulting in the activation of a downstream protein kinase (49).

Surprising illustrations of the complexity and importance of the organization of redox signaling and the control of the oxidation state of cysteines are arising at an increasing rate. The Fanconi anemia group $\mathrm{C}$ protein protects the survival of hematopoietic progenitor cells. The function of this protein in hematopoietic cells is to interact with glutathione-Stransferase P1 (50). The Fanconi anemia group C protein lacks homology with conventional disulfide reductases; however, it functions by preventing the oxidation of cysteines and the formation of inactivating disulfide bonds within glutathione-S-transferase P1 during apoptosis, allowing the enzyme to function in its protective role.

Not surprisingly, with the extension of redox signaling into all aspects of cell function, the enzymes and proteins in these pathways are emerging as potential drug targets. In the final article in this series, Richard Jack will describe an approach to the development, and potential clinical use, of novel small-molecule bi-ligand inhibitors of bacterial and mammalian oxidoreductases from chemical libraries that were specifically developed using NADs as templates (51).

Each of the areas described above is represented by an in-depth review in this Perspective series. It is hoped that they will stimulate readers to consider the role of signaling by redox signaling and oxygen in a broad context that perhaps touches on their own work.

\section{Acknowledgments}

The author would like to thank Lawrence Marnett, Carl Nathan, John Kyriakis, and Bettie Sue Masters for their helpful advice and insight during preparation of this manuscript. The work was supported by NIH grant R01GM-061823 and an unrestricted gift from the Jewish Communal Fund.

\footnotetext{
1. Baehner, R.L., and Karnovsky, M.L. 1968. Deficiency of reduced nicotinamide-adenine dinucleotide oxidase in chronic granulomatous disease. Science. 162:1277-1279.

2. Baehner, R.L., Gilman, N., and Karnovsky, M.L. 1970. Respiration and glucose oxidation in human and guinea pig leukocytes: comparative studies. J. Clin. Invest. 49:692-700.
} 
3. Baehner, R.L., Nathan, D.G., and Karnovsky, M.L. 1970. Correction of metabolic deficiencies in the leukocytes of patients with chronic granulomatous disease. J. Clin. Invest. 49:865-870.

4. Semenza, G.L. 2001. HIF-1, $\mathrm{O}_{2}$, and the 3 PHDs: how animal cells signal hypoxia to the nucleus. Cell. 107:1-3.

5. Semenza, G.L. 1999. Perspectives on oxygen sensing. Cell. 98:281-284. 6. Semenza, G.L. 2000. HIF-1 and human disease: one highly involved factor. Genes Dev. 14:1983-1991.

7. Bruick, R.K., and McKnight, S.L. 2001. A conserved family of prolyl-4hydroxylases that modify HIF. Science. 294:1337-1340.

8. Min, J.-H., et al. 2002. Structure of an HIF-1-pVHL complex: hydroxyproline recognition in signaling. Science. 296:1886-1889.

9. Ivan, M., et al. 2001. HIF targeted for VHL-mediated destruction by proline hydroxylation: implications for $\mathrm{O}_{2}$ sensing. Science. 292:464-468.

10. Lando, D., Peet, D.J., Whelan, D.A., Gorman, J.J., and Whitelaw, M.L. 2002. Asparagine hydroxylation of the HIF transactivation domain: a hypoxic switch. Science. 295:858-861.

11. Safran, M., and Kaelin, W.G. 2003. HIF hydroxylation and the mammalian oxygen-sensing pathway. J. Clin. Invest. In press.

12. Rutter, J., Reick, M., Leeju, C., Wu, L.C., and McKnight, S.L. 2001. Regulation of clock and NPAS2 DNA binding by the redox state of NAD cofactors. Science. 293:510-514.

13. Reick, M., Garcia, J.A., Dudley, C., and McKnight, S.L. 2001. NPAS2: an analog of clock operative in the mammalian forebrain. Science. 293:506-509.

14. Zhang, Q., Piston, D.W., and Goodman, R.H. 2001. Regulation of corepressor function by nuclear NADH. Science. 295:1895-1897.

15. Kim, S.O., et al. 2002. OxyR: a molecular code for redox-related signaling. Cell. 109:383-396.

16. Stamler, J.S., Lamas, L., Ferric, C., and Fang, F.C. 2001. Nitrosylation: the prototypic redox-based signaling mechanism. Cell. 106:675-683.

17. Smith, W.L., and Lands, W.E. 1972. Oxygenation of polyunsaturated fatty acids during prostaglandin biosynthesis by sheep vesicular gland. Biochemistry. 11:3276-3285.

18. Smith, W.L., and Lands, W.E. 1972. Oxygenation of unsaturated fatty acids by soybean lipoxygenase. J. Biol. Chem. 247:1038-1047.

19. Smith, W.L., Garavito, R.M., and DeWitt, D.L. 1996. Prostaglandin endoperoxide $\mathrm{H}$ synthases (cyclooxygenases)-1 and -2. J. Biol. Chem. 271:33157-33160.

20. FitzGerald, G.A., and Loll, P. 2001. COX in a crystal ball: current status and future promise of prostaglandin research. J. Clin. Invest. 107:1335-1338.

21. Brash, A.R. 2001. Arachidonic acid as a bioactive molecule. J. Clin. Invest. 107:1339-1345.

22. Fitzpatrick, F.A., and Soberman, R.J. 2001. Regulated formation of eicosanoids. J. Clin. Invest. 107:1347-1351.

23. Serhan, C.N., and Oliw, E. 2001. Unorthodox routes to prostanoid formation: new twists in cyclooxygenase-initiated pathways. J. Clin. Invest. 107:1481-1489.

24. Smith, W.L., and Langenbach, R. 2001. Why there are two cyclooxygenase isozymes. J. Clin. Invest. 107:1491-1495.

25. Patrono, C., Patrignani, P., and García Rodríguez, L.A. 2001. Cyclooxygenase-selective inhibition of prostanoid formation: transducing biochemical selectivity into clinical read-outs. J. Clin. Invest. 108:7-13. doi:10.1172/JCI200113418

26. Tilley, S.L., Coffman, T.M., and Koller, B.H. 2001. Mixed messages: modulation of inflammation and immune responses by prostaglandins and thromboxanes. J. Clin. Invest. 108:15-23. doi:10.1172/JCI200113416.

27. Narumiya, S., and FitzGerald, G.A. 2001. Genetic and pharmacological analysis of prostanoid receptor function. J. Clin. Invest. 108:25-30. doi:10.1172/JCI200113455

28. Soberman, R.J., and Christmas, P. 2003. The organization and consequences of eicosanoid signaling. J. Clin. Invest. In press.
29. Lipton, S.A., and Bossy-Wetzel, E. 2002. Dueling activities of AIF in cell death versus survival: DNA binding and redox activity. Cell. 111:147-150

30. Klein, J.A., et al. 2002. The harlequin mouse mutation downregulates apoptosis-inducing factor. Nature. 419:367-374.

31. Klein, J., and Ackerman, S.L. 2003. Oxidative stress, cell cycle, and neurodegeneration. J. Clin. Invest. In press.

32. Kumar, V., et al. 2002. Transcription corepressor CtBP is an $\mathrm{NAD}^{+}$-regulated dehydrogenase. Mol. Cell. 10:857-869.

33. Marnett, L.J., Riggins, J.N., and West, J.D. 2003. Endogenous generation of reactive oxidants and electrophiles and their reactions with DNA and protein. J. Clin. Invest. 111:583-593. doi:10.1172/JCI200318002.

34. Moos, P.J., Edes, K., and Fitzpatrick, F.A. 2000. Inactivation of wild-type p53 tumor suppressor by electrophilic prostaglandins. Proc. Natl. Acad. Sci. U. S. A. 97:9215-9220.

35. Hildeman, D.A., Mitchell, T., Kappler, J., and Marrack, P. 2003. T cell apopotosis and reactive oxygen species. J. Clin. Invest. 111:576-581. doi:10.1172/JCI200318007.

36. Nathan, C. 2003. Specificity of a third kind: reactive oxygen and nitrogen intermediates in cell signaling. J. Clin. Invest. In press.

37. Rhee, S.G., Bae,Y.S., Lee, S.-R., and Kwon, J. 2000. Hydrogen peroxide: a key messenger that modulates protein phosphorylation through cysteine oxidation. Sci. STKE. October 10. PE1.

38. Sundaresan, M., Yu, Z.-X., Ferrans, V.J., Irani, K., and Finkel, T. 1995. Requirement for generation of $\mathrm{H}_{2} \mathrm{O}_{2}$ for platelet-derived growth factor signal transduction. Science. 270:296-299.

39. May, J.M., and de Haen, C. 1979. Insulin-stimulated intracellular hydrogen peroxide production in rat epididymal fat cells. J. Biol. Chem 254:2214-2220.

40. Woo, C.-H., et al. 2002. Leukotriene $B_{4}$ stimulates rac-ERK cascade to generate reactive oxygen species that mediates chemotaxis. J. Biol. Chem. 277:8572-8578.

41. Denu, J.M., and Dixon, J.E. 1998. Protein tyrosine phosphatases: mechanisms of catalysis and regulation. Curr. Opin. Chem. Biol. 2:633-641.

42. Lee, S.R., Kwon, K.S., Kim, S.R., and Rhee, S.G. 1998. Reversible inactivation of protein-tyrosine phosphatase $1 \mathrm{~B}$ in A431 cells stimulated with epidermal growth factor. J. Biol. Chem. 273:15366-15372.

43. Denu, J.M., and Tanner, K.G. 1998. Specific and reversible inactivation of protein tyrosine phosphatases by hydrogen peroxide: evidence for a sulfenic acid intermediate and implications for redox regulation. Biochemistry. 37:5633-5642.

44. Fetrow, J.S., Siew, N., and Skolnick, J. 1999. Structure-based functional motif identifies a potential disulfide oxidoreductase active site in the serine/threonine protein phosphatase-1 subfamily. FASEB J. 13:1866-1874.

45. Saitoh, M., et al. 1998. Mammalian thioredoxin is a direct inhibitor of apoptosis signal-regulating kinase (ASK)1. EMBO J. 17:2596-2600.

46. Liu, H., Nishitoh, H., Ichijo, H., and Kyriakis, J.M. 2000. Activation of apoptosis signal-regulating kinase 1 (ASK1) by tumor necrosis factor receptor-associated factor 2 requires prior dissociation of the ASK1 inhibitor thioredoxin. Mol. Cell. Biol. 20:2198-2208.

47. Arunachalam, B., Phan, U.T., Geuze, H.J., and Cresswell, P. 2000. Enzymatic reduction of disulfide bonds in lysosomes: characterization of a gamma-interferon-inducible lysosomal thiol reductase (GILT). Proc. Natl. Acad. Sci. U. S. A. 97:745-750.

48. Phan, U.T., Arunachalam, B., and Cresswell, P. 2000. Gamma-interferoninducible lysosomal thiol reductase (GILT): maturation, activity and mechanism of action. J. Biol. Chem. 275:25907-25914.

49. Lander, H.M., et al. 1996. Redox regulation of cell signalling. Nature. 381:380-381.

50. Cumming, R.C., et al. 2001. Fanconi anemia group C protein prevents apoptosis in hematopoietic cells through redox regulation of GSTP1. Nat. Med. 7:814-820.

51. Jack, R.M. 2003. Drug design based on oxidation-reduction. J. Clin. Invest. In press. 